\title{
Trial of Oral Metoclopramide on Diurnal Bruxism of Brain Injury
}

\author{
Ho Sung Yi, $\mathrm{MD}^{1}$, Hyoung Seop Kim, $\mathrm{MD}^{2}$, Mi Ri Seo, $\mathrm{MD}^{1}$
}

\begin{abstract}
${ }^{1}$ Department of Rehabilitation Medicine, Yonsei University College of Medicine, Seoul; ${ }^{2}$ Department of Physical Medicine and Rehabilitation, National Health Insurance Corporation Ilsan Hospital, Goyang, Korea
\end{abstract}

Bruxism is a diurnal or nocturnal parafunctional activity that includes tooth clenching, bracing, gnashing, and grinding. The dopaminergic system seems to be the key pathophysiology of bruxism and diminution of dopaminergic transmission at the prefrontal cortex seems to induce it. We report two patients with diurnal bruxism in whom a bilateral frontal lobe injury resulted from hemorrhagic stroke or traumatic brain injury. These patients' bruxism was refractory to bromocriptine but responded to low-dose metoclopramide therapy. We propose that administering low doses of metoclopramide is possibly a sound method for treating bruxism in a brain injury patient with frontal lobe hypoperfusion on positron emission tomography imaging.

Keywords Bruxism, Dopamine receptors, Metoclopramide

\section{INTRODUCTION}

Bruxism is a diurnal or nocturnal parafunctional activity that includes clenching, bracing, gnashing, and grinding of the teeth. Its prevalence is reported to be $20 \%$ among the adult population [1]. Previous studies have pointed out alterations in central neurotransmission, particularly dopaminergic neurotransmission, as the main cause of bruxism. Recent studies suggest that hypersensitivity of the dopamine receptors is associated with bruxism [2]. We report two patients with diurnal

Received April 13, 2012; Accepted June 21, 2012

Corresponding author: Hyoung Seop Kim

Department of Physical Medicine and Rehabilitation, National Health Insurance Corporation Ilsan Hospital, 100 Ilsan-ro, Ilsandong-gu, Goyang 410-719, Korea

Tel: +82-31-900-0137, Fax: +82-31-900-0343, E-mail: rekhs@nhimc.or.kr

(c) This is an open-access article distributed under the terms of the Creative Commons Attribution Non-Commercial License (http://creativecommons. org/licenses/by-nc/3.0) which permits unrestricted noncommercial use, distribution, and reproduction in any medium, provided the original work is properly cited.

Copyright $\odot 2013$ by Korean Academy of Rehabilitation Medicine bruxism in whom bilateral frontal lobe hypoperfusion resulted from hemorrhagic stroke or traumatic brain injury. These patients' bruxism was refractory to bromocriptine but responded to metoclopramide therapy. These cases suggest that blockade of the hypersensitive presynaptic dopaminergic receptor can reduce bruxism.

\section{CASE REPORTS}

\section{Case 1}

A 51-year-old man was admitted for subarachnoid hemorrhage caused by a rupture of the left anterior communicating artery aneurysm. After acute treatment for a month, he was transferred to the department of rehabilitation for intensive therapy. Physical and neurological examinations were done. The score of the Korean version of the Mini-Mental Status Examination (K-MMSE) was 16. There were no abnormal findings on cranial nerve function tests, and no definite extraocular movement limitations or facial palsies were noted. Manual muscle power test showed that the Medical Research Council (MRC) 
grade of both the upper and lower extremities was grade II and I, respectively. The patient had been suffering from teeth grinding and jaw clenching, which had begun 3 weeks after the onset of a cerebral hemorrhage. Dystonia of the tongue, face or any other part of the body was absent. This symptom was aggravated during the daytime, especially during meals, and subsided during sleep. Teeth grinding worsened as time went on. An injection of botulinum toxin was given 5 weeks after the onset of bruxism; 40 units to each side of the bilateral masseter, 15 units to each side of the bilateral lateral pterygoid, and 15 units to each side of the bilateral medial pterygoid muscles. The symptom was followed up after 2 weeks, and the injected botulinum toxin showed no effect. His bruxism was also refractory to bromocriptine therapy. Thus, a $10 \mathrm{mg}$ intramuscular metoclopramide $\mathrm{HCl}$ injection was administered for treatment of the bruxism. The bruxism subsided dramatically 5 hours after the injection and did not recur for the subsequent 12 hours. Oral metoclopramide medication in dosages of $15 \mathrm{mg}$ per day was initiated the next day, which successfully abolished his bruxism after 2 days. Bruxism did not recur during the metoclopramide therapy. Magnetic resonance imaging of the brain showed a subacute intracranial hemorrhage at the left basal frontal lobe (Fig. 1). Positron emission tomography (PET) also showed a metabolic defect at the left basal frontal lobe, along with diffuse hypometabolism at the bilateral frontal, temporal, and parietal cortexes. A blink reflex study and electroencephalography (EEG) were normal. Transcranial and carotid Doppler were also normal. Anticholinergic agents, which are known to decrease the effectiveness of metoclopramide, were not administered for any reason. Any drug that induced bruxism or decreased the effect of levodopa was not administered either.

\section{Case 2}

A 36-year-old male, who had no significant medical history nor neurologic abnormalities, was involved in a motorcycle accident. He was admitted to the emergency room unconscious, and after being diagnosed with bilateral frontal and right temporal intracranial hemorrhage (Fig. 2), he underwent craniectomy and hematoma evacuation. Two years after the onset of the injury, he was transferred to the department of rehabilitation for intensive therapy. The K-MMSE score at the time was 15. A manual muscle power test showed that the MRCs of both the upper and lower extremities of the right side were grade IV, and those of the upper and lower extremities of the left side were grade II and I, respectively. He had suffered from teeth grinding and jaw clenching for 2

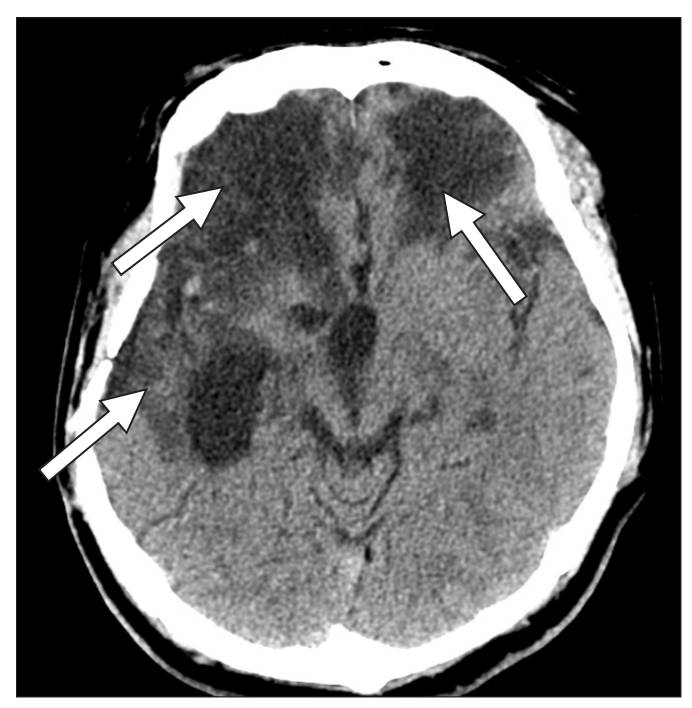

Fig. 2. A cranial computerized tomography scan from case 2 , showing extensive encephalomalacia at the bilateral frontal lobes and the right temporal lobe from previous trauma (arrows).

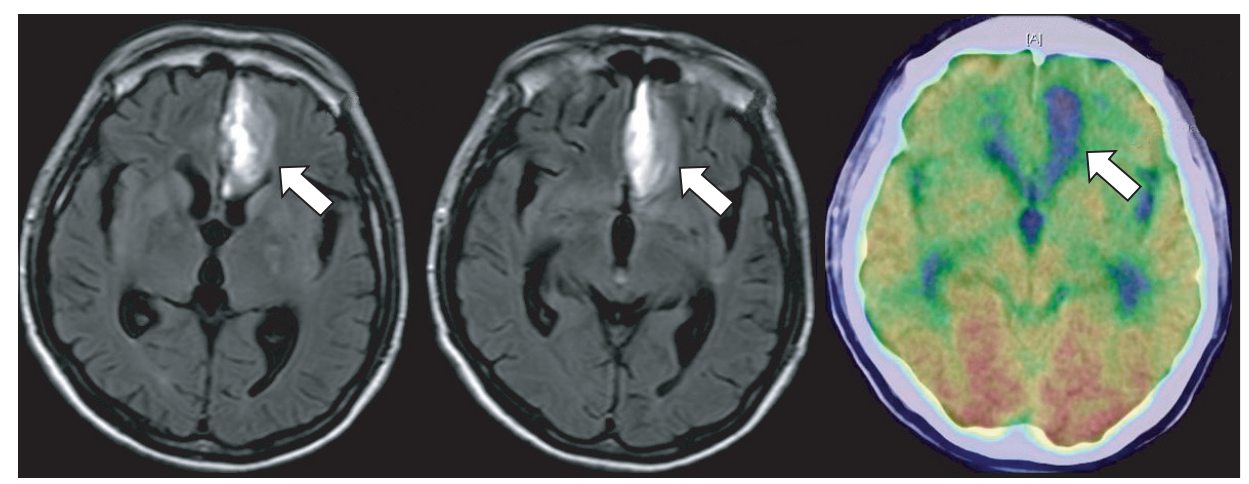

872
Fig. 1. Magnetic resonance imaging of the brain from case 1, showing subacute intracranial hemorrhage in the left basal frontal lobe (arrows). Positron emission tomography also shows metabolic defects in the left basal frontal lobe along with diffuse hypometabolism in the bilateral frontal, temporal, and parietal cortex. 
years but had not received any treatment. The bruxism occurred during the daytime and subsided during sleep. There was no dystonia of the tongue, face or elsewhere. Bruxism was refractory to bromocriptine therapy. Oral metoclopramide administration of $9 \mathrm{mg}$ per day was started to treat the bruxism, and it improved dramatically three days after the initiation of the medication. Bruxism did not recur during the course of the metoclopramide therapy. A cranial computerized tomography scan revealed extensive encephalomalacia in the bilateral frontal lobes and the right temporal lobe from the previous trauma. The EEG was normal. The patient also did not take any medication that may have induced bruxism or decreased the effects of the metoclopramide or levodopa.

\section{DISCUSSION}

The pathogenesis of bruxism continues to be a matter of scientific debate, and different theories have been proposed over the years. Previous studies revealed that individuals with sleep bruxism have higher levels of urinary catecholamines including dopamine [3]. Bruxism may develop after exposure to haloperidol or selective serotonin reuptake inhibitors (SSRIs) [3], or in untreated schizophrenia [4]. These studies suggested that the central dopaminergic system may be of some importance in explaining the origin of bruxism.

The central role of the frontal lobe in bruxism is supported by a previous study [5]. Our patients had a bilateral frontal lobe injury that resulted from a hemorrhagic stroke or traumatic brain injury. Although dopamine receptors are widely distributed in the brain, different areas have different receptor types and densities, presumably reflecting different functional roles in different areas. The mesocortical pathway, which is a neural pathway that connects the ventral tegmentum to the frontal cortex, modulates the motor output of the prefrontal cortex by maintaining an inhibitory signal [6].

The acute (short-term) use of L-dopa, a dopamine precursor and a precursor of bromocriptine, a dopamine receptor agonist, inhibits bruxism activity in controlled polysomnographical studies [7]. Meanwhile, medications that exert an indirect influence on the dopaminergic system, like selective serotonin reuptake inhibitors or dopaminoceptor antagonists, are known to induce bruxism [2]. These studies suggest that bruxism may be induced by diminution of dopaminergic transmission at the prefrontal cortex.

But interestingly, our patients showed another interaction of neurochemistry in diurnal bruxism. Our patients were refractory to bromocriptine, but responded well to metoclopramide, a selective dopamine receptor antagonist. This can be explained by the preferential action of metoclopramide on presynaptic dopamine receptors. While a high dose of metoclopramide is known to block postsynaptic dopamine receptors, a low dose of metoclopramide is more potent in blocking dopamine receptors of the dopamine nerve terminals (presynaptic dopamine receptors) [8]. It results in a facilitated release of dopamine without a blockade of the postsynaptic dopamine receptors [8]. Therefore, a selective blockade of the hypersensitive presynaptic dopamine receptors by low-dose metoclopramide administration recovers the dopaminergic flow and attenuates bruxism. On the other hand, L-dopa and bromocriptine activate both the presynaptic and postsynaptic dopamine receptors with a net effect, resulting in no changes in dopaminergic innervations [2].

Chen et al. [2] reported a subjective reduction of bruxism activity observed in three patients receiving dopamine receptor antagonist medication. The patients had left frontal hypoperfusion on imaging and showed no effect to the previous administration of either L-dopa or bromocriptine. The reduction of bruxism was associated with a reperfusion of the prefrontal cortex during waketime. It has been suggested that in patients with both awake and sleep bruxism, a different mechanism may operate in the genesis of bruxism [2].

Drug use, such as SSRIs, methylenedioxymethamphetamine, methylenedioxyamphetamine, methylphenidate, and other amphetamines may cause bruxism [9]. The concurrent administration of anticholinergic drugs, pramlintide, for example, is known to decrease the effectiveness of metoclopramide, and such medications were not administered in our cases [10]. There was also no administration of drugs that may interfere with the actions of levodopa, such as haloperidol, loxapine, phenothiazines, in our cases.

In an animal study, $0.3 \mathrm{mg} / \mathrm{kg}$ of metoclopramide given intraperitoneally facilitated the release of dopamine. A dose ten times higher did not block presynaptic dopamine receptors but seemed to block postsynaptic 
dopamine receptors, judging from the inhibition of the apomorphine-induced turning [8]. The proper metoclopramide dosage for bruxism might be the usual adult dose for gastroparesis, depending upon the symptoms being treated and the clinical response. The usual dose of metoclopramide for treating gastroparesis is 10 to $15 \mathrm{mg}$ up to 3 times a day orally.

In this study, we report two patients with bruxism in whom bilateral frontal lobe lesions resulted from an acquired brain injury. These patients' bruxism was refractory to bromocriptine but responded to low-dose metoclopramide therapy. Our cases indicate that administering a low dose of metoclopramide is possibly a sound method for treating bruxism in a brain injury patient with frontal lobe hypoperfusion on a PET image. Further study is needed under blind and controlled conditions using various tools to characterize the symptoms of bruxism, such as an EMG and audio/video or polysomnographic assessments.

\section{CONFLICT OF INTEREST}

No potential conflict of interest relevant to this article was reported.

\section{REFERENCES}

1. Lavigne GJ, Khoury S, Abe S, Yamaguchi T, Raphael K. Bruxism physiology and pathology: an overview for clinicians. J Oral Rehabil 2008;35:476-94.

2. Chen WH, Lu YC, Lui CC, Liu JS. A proposed mecha- nism for diurnal/nocturnal bruxism: hypersensitivity of presynaptic dopamine receptors in the frontal lobe. J Clin Neurosci 2005;12:161-3.

3. Seraidarian P, Seraidarian PI, das Neves Cavalcanti B, Marchini L, Claro Neves AC. Urinary levels of catecholamines among individuals with and without sleep bruxism. Sleep Breath 2009;13:85-8.

4. Bannon MJ, Roth RH. Pharmacology of mesocortical dopamine neurons. Pharmacol Rev 1983;35:53-68.

5. Areso MP, Giralt MT, Sainz B, Prieto M, Garcia-Vallejo P, Gomez FM. Occlusal disharmonies modulate central catecholaminergic activity in the rat. J Dent Res 1999;78:1204-13.

6. Arnsten AF. Catecholamine regulation of the prefrontal cortex. J Psychopharmacol 1997;11:151-62.

7. Lobbezoo F, Lavigne GJ, Tanguay R, Montplaisir JY. The effect of catecholamine precursor L-dopa on sleep bruxism: a controlled clinical trial. Mov Disord 1997;12:73-8.

8. Alander T, Anden NE, Grabowska-Anden M. Metoclopramide and sulpiride as selective blocking agents of pre- and postsynaptic dopamine receptors. Naunyn Schmiedebergs Arch Pharmacol 1980;312:145-50.

9. Winocur E, Gavish A, Voikovitch M, Emodi-Perlman A, Eli I. Drugs and bruxism: a critical review. J Orofac Pain 2003;17:99-111.

10. Brock-Utne JG, Rubin J, Downing JW, Dimopoulos GE, Moshal MG, Naicker M. The administration of metoclopramide with atropine: a drug interaction effect on the gastro-oesophageal sphincter in man. Anaesthesia 1976;31:1186-90. 